\title{
Building a Neural Machine Translation System
}

\author{
Stefanie Dinman, William Jin
}

\section{Method}

\subsection{Neural Machine Translation}

Neural Machine Translation (NMT) is an end-to-end structure which could directly model the translation probability between a source sentence $\mathbf{x}=x_{1}, \ldots, x_{J}$ and a target sentence $\mathbf{y}=y_{1}, \ldots, y_{I}$ word by word:

$$
P(\mathbf{y} \mid \mathbf{x})=\prod_{i=1}^{I} P\left(y_{i} \mid \mathbf{y}_{<i}, \mathbf{x} ; \theta\right)
$$

where $\mathbf{y}_{<i}$ is the partial translation before decoding step $i$ and $\theta$ is parameters of the NMT. The probability of generating the $i$-th word $P\left(y_{i} \mid y_{<i}, \mathbf{x} ; \theta\right)$ is calculated by

$$
P\left(y_{i} \mid \mathbf{y}_{<i}, \mathbf{x} ; \theta\right) \propto \exp \left\{f\left(y_{i-1}, \mathbf{s}_{i}, \mathbf{c}_{i} ; \theta\right)\right\}
$$

where $\mathbf{s}_{i}$ is the $i$-th hidden state of the decoder and $f(\cdot)$ is a non-linear activation function of the decoder state. $\mathbf{c}_{i}$ is a distinct source representation for time $i$, calculated as a weighted sum of the source annotations: $\mathbf{c}_{i}=\sum_{j=1}^{J} \alpha_{i, j} \cdot \mathbf{h}_{j}$, where $\mathbf{h}_{j}$ is the annotation of $x_{j}$ from a encoder, and its weight $\alpha_{i, j}$ is computed by

$$
\alpha_{i, j}=\frac{\exp \left(e_{i, j}\right)}{\sum_{j^{\prime}=1}^{J} \exp \left(e_{i, j^{\prime}}\right)} \text { with } e_{i, j}=a\left(\mathbf{s}_{i-1}, \mathbf{h}_{j}\right)
$$

where $a(\cdot)$ is an attention model that scores how well $y_{i}$ and $\mathbf{h}_{j}$ (i.e. $x_{j}$ ) match. The encoder and decoder can be implemented as Recurrent Neural Network (RNN) [1], Convolutional Neural Network (CNN) [3], or Self-Attention Network (SAN) [11]. In this assignment, RNN-based NMT is called RNNSEARCH.

The parameters of the NMT $\theta$ are trained to maximize the likelihood of training instances $\left\{\left[x^{n}, y^{n}\right]\right\}_{n=1}^{N}$ :

$$
L(\theta)=\underset{\theta}{\arg \max } \sum_{n=1}^{N} \log P\left(y^{n} \mid x^{n} ; \theta\right)
$$

\subsection{Mixture of Softmaxes (MoS)}

Mixture of Softmaxes (MoS) [12] is introduced to address the expressiveness limitations of Softmax-based models and (author?) [7] applied that to the NMT. 


\begin{tabular}{c|c|c|c|c}
\hline$\#$ & Vocabulary & Input-feeding & Dev BLEU & Test BLEU \\
\hline 1 & \multirow{2}{*}{ Fixed Vocabulary } & Yes & 29.64 & 27.60 \\
2 & & No & 29.12 & 27.25 \\
\hline 3 & \multirow{2}{*}{ Open Vocabulary } & Yes & 30.51 & $\mathbf{2 8 . 7 1}$ \\
4 & & No & 30.02 & 28.31 \\
\hline
\end{tabular}

Table 1: Performance of models with or without input-feeding approach. We set embedding size and hidden size to be 256 , and use a 1 layer LSTM model, which is the same as the provided based line by our Teaching Assistant.

More details about expressiveness limitations of Softmax-based models and motivation of MoS could be found in [12] and we just describe the implementation of MoS here.

MoS To tackle the Softmax bottleneck problem, MoS formulates the distribution as the weighted average of $K$ Softmax components:

$$
P_{\theta}(x \mid c)=\sum_{k=1}^{K} \pi_{c, k} \frac{\exp \mathbf{h}_{c, k}^{\top} \mathbf{w}_{x}}{\sum_{x^{\prime}} \exp \mathbf{h}_{c, k}^{\top} \mathbf{w}_{x^{\prime}}}
$$

where $\pi_{c, k}$ is the mixture weight of the $k$-th Softmax component and $\mathbf{h}_{c, k}$ is the $k$-th context vector. On language modeling, it has been shown empirically that such a formulation leads to a high rank matrix. Note that since all Softmaxes share the same word embedding matrix, the number of parameters do not increase rapidly with more mixtures, preventing overfitting.

\section{Experiment}

\subsection{Dataset}

German $\rightarrow$ English $(\mathrm{DE} \rightarrow \mathrm{EN})$ dataset from IWSLT 2014 evaluation campaign [2] is employed and sentences longer than 50 words are removed. Finally, the dataset consists of about $153 k, 7 k$ and $7 k$ sentences in training, dev and test sets respectively.

We use two methods to build the vocabulary:

- Fixed Vocabulary We replace words which just occur once in the training data by a special token ' $\langle u n k\rangle$ ' and keep frequent words. Finally, there are 32011 and 22824 most frequent words and specially tokens in German and English corpora respectively.

- Open Vocabulary We employ Byte Pair Encoding (BPE) $[10,6]$ with $30 k$ merge operations for each language separately. 


\subsection{RNNSearch}

Architecture wise, we employ a seq2seq model with dot-product attention $[1,8]$ as our RNNSEARCH baseline model. Specially, the encoder is a bidirectional LSTM [4] with each direction being size 128 and the decoder is another LSTM with size 256. Furthermore, we employ input feeding technique $[8,5]$ in our NMT systems. More implementation details could be found in Section 2.4. Generally, there are several key hyper-parameters which could affect the performance of RNNSEARCH model, such as the number of layers in the encoder and decoder, the hidden size of each LSTM, etc. Therefore, in this assignment, we aim to explore these hyper-parameters.

Input-feeding Approach In [8], input-feeding technique is proposed to add previous attention vectors into the inputs at the next time step. Therefore, the model could be aware of previous alignment decisions. In order to investigate the effect of input feeding, we remove it from out baseline models with fixed and open vocabularies and the results are shown in Table 1. We could find that models with input-feeding is slightly better than its counterpart without inputfeeding because models with input-feeding could make the alignment decision with previous alignment information.

Fixed Vocabulary vs Open Vocabulary We employ two methods to build the vocabulary which are described in Section 2.1. From the Rows 1 vs. 5, 2 vs 6,3 vs 7, 4 vs 8 in Table. 2, we could find that open vocabulary-based NMT systems outperform their fixed vocabulary-based counterparts. We ascribe this fact to that the open vocabulary NMT system could handle out-of-vocabulary cases.

The number of layers The number of layers in the encoder and decoder could affect the performance. From the Rows 1 vs. 3,2 vs 4,5 vs 7,6 vs 8 in Table. 2, NMT systems with two layer stacked-LSTM in the encoder and decoder outperform their counterparts with just one layer. We attribute this to the fact that more layers could improve the capacity and generalization ability of the NMT system.

The number of units in LSTM In this experiment, we would like to explore NMT systems with different number of units in LSTM. From the Rows 1 vs. 2,3 vs 4,5 vs 6,7 vs 8 in Table 2, it is clear to find that NMT systems with larger number of units could achieve better performance. With more units in LSTM, it could improve its ability to "remeber" longer and more complicated sequences.

\section{$2.3 \quad$ RNNSearch + MoS}

Due to the need of computing multiple Softmaxes, a model with the Mixture of Softmaxes layer needs large memory. Therefore, given the memory of one GPU 


\begin{tabular}{c|c|c|c|c|c|c}
\hline$\#$ & Vocabulary & Emb. Size & Hidden Size & \# Layer & Dev BLEU & Test BLEU \\
\hline 1 & & 256 & 256 & 1 & 29.64 & 27.60 \\
2 & \multirow{3}{*}{ Fixed Voc } & 256 & 512 & 1 & 29.98 & 28.02 \\
3 & 256 & 256 & 2 & 30.31 & 28.63 \\
4 & & 256 & 512 & 2 & 30.68 & 29.08 \\
\hline 5 & & 256 & 256 & 1 & 30.51 & 28.71 \\
6 & \multirow{2}{*}{ BPE } & 256 & 512 & 1 & 31.23 & 29.44 \\
7 & & 256 & 256 & 2 & 31.13 & 29.49 \\
8 & & 256 & 512 & 2 & 31.89 & 30.30 \\
9 & & 512 & 512 & 2 & 32.28 & $\mathbf{3 0 . 6 3}$ \\
\hline
\end{tabular}

Table 2: Performances of NMT systems with different architectures. By adding more parameters (increasing embedding size, hidden size or number of layers), the performance of the model consistentlt improves.

\begin{tabular}{c|c|c|c|c}
\hline$\#$ & \# Mixtures & Memory (GB) & Dev BLEU & Test BLEU \\
\hline 1 & 1 & 5.54 & 29.64 & 27.60 \\
2 & 3 & 10.84 & 30.17 & 28.15 \\
\hline
\end{tabular}

Table 3: Comparison of RNNSEARCH and RNNSEARCH+MoS models.

\begin{tabular}{c|c|c|c|c}
\hline Model & BLEU (1:15) & BLEU (11:30) & BLEU (31:45) & BLEU (>45) \\
\hline RNNSEARCH & 29.05 & 28.6 & 27.1 & 22.95 \\
\hline RNNSEARCH+MoS & 29.14 & 28.72 & 27.77 & 25.08 \\
\hline
\end{tabular}

Table 4: Comparison of RNNSEARCH and RNNSEARCH+MoS models by BLEU scores with respect to the source sentence lengths. 


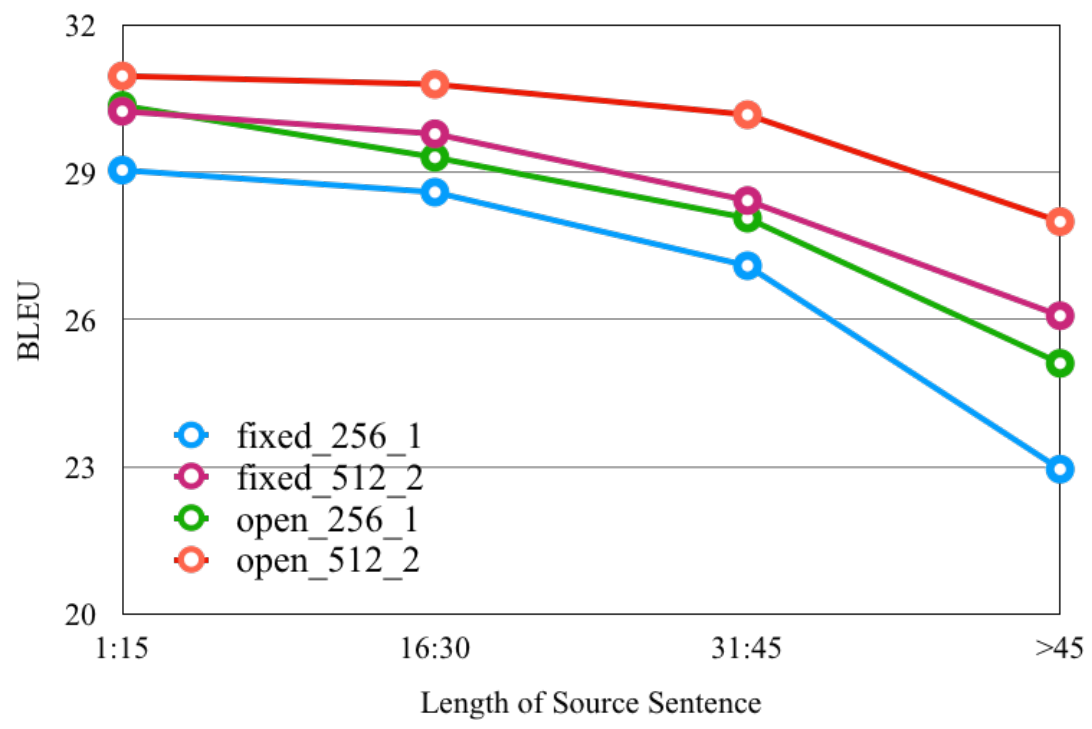

Figure 1: BLEU of translations with respect to the source sentence lengths. "fixed" and "open" denote two vocabulary building methods. "256" and "512" show the hidden size of the NMT systems. "1" and "2" indicate the number of layers in the encoder and decoder.

as the budget, in this experiment, we only add MoS layer into our baseline models with the fixed vocabulary. Note that while open vocabulary-based models have a smaller vocabulary, due to the longer sentence which is caused by BPE splitting, the memory is still larger than that of fixed-vocabulary models. The result is shown in Table 3. From this table, it is clear to find that the MoS-based NMT models outperform original NMT models, which indicates that MoS could improve the generalization ability of NMT models. However, MoS will require a much larger memory so that the memory-reduction methods are necessary to be employed for larger mdoels.

\subsection{Implementation details}

The batch size is 64 , the embedding dimension is 256 and stochastic gradient descent is employed to train our models. Specifically, the initial learning for all RNNSEARCH models is 1.0 and the learning rate stays the same for the first eight warm-up epochs, after which the learning rate is droped by half every epoch. For the RNNSEARCH+MoS model, the initial learning rate is 0.8 and learning decay is 0.8 .

The corpus-level BLEU score [9] is employed to evaluate the performance of different NTM systems. 


\subsection{Length Analysis}

We group test sentences of similar lengths and compute the BLEU score for each group and the result is shown in Fig.1. Specially, the four length spans contain 3424, 2346, 686 and 294 sentences respectively. Given a single mode, we could find in this figure that BLEU of shorter sentence group is higher than that of longer one. Intuitively, this is because that shorter sentences are easier to be generated than longer ones.

Furthermore, models with more hidden units or layers in the NMT system could improve the BLEU scores of all group especially the longer sentence. We attribute this fact to that with more hidden units or layers, the NMT system could translate longer sentences much better and the NMT system with less hidden units or layers is already able to translate shorter sentences well.

We also calculate the BLEU scores for different length of source sentence groups of RNNSEARCH and RNNSEARCH+MOS models and the result is shown in Table 4. Intuitively, we could find that RNNSEARCH+MoS model is capable to translate longer sentences (especially source sentences longer than 45 tokens) better than RNNSEARCH model, which means that the MoS layer helps RNNSEARCH model improve the expressiveness of the softmax layer. For the shorter sentences, the the BLEU score of RNNSEARCH model is similar to that of RNNSEARCH+MoS model. This is because that the original softmax layer have already enough capacity to handle these shorter sentences.

\section{Conclusion}

In this assignment, we implement and analyze Neural Machine Translation (NMT) systems with different configurations and find that with more layers and number of hidden unit, NMT models could achieve better performances. Furthermore, we incorporate Mixture of Softmaxes (MoS) [12] into the NMT system and the result shows that MoS could improve its generalization ability.

\section{References}

[1] Dzmitry Bahdanau, Kyunghyun Cho, and Yoshua Bengio. Neural machine translation by jointly learning to align and translate. arXiv preprint arXiv:1409.0473, 2014.

[2] Mauro Cettolo, Jan Niehues, Sebastian Stüker, Luisa Bentivogli, and Marcello Federico. Report on the 11th iwslt evaluation campaign, iwslt 2014. In Proceedings of the International Workshop on Spoken Language Translation, Hanoi, Vietnam, 2014.

[3] Jonas Gehring, Michael Auli, David Grangier, Denis Yarats, and Yann N Dauphin. Convolutional sequence to sequence learning. arXiv preprint arXiv:1705.03122, 2017. 
[4] Sepp Hochreiter and Jürgen Schmidhuber. Long short-term memory. Neural computation, 9(8):1735-1780, 1997.

[5] Xiang Kong, Jeung-Yoon Choi, and Stefanie Shattuck-Hufnagel. Evaluating automatic speech recognition systems in comparison with human perception results using distinctive feature measures. In 2017 IEEE International Conference on Acoustics, Speech and Signal Processing (ICASSP), pages 5810-5814. IEEE, 2017.

[6] Xiang Kong, Zhaopeng Tu, Shuming Shi, Eduard Hovy, and Tong Zhang. Neural machine translation with adequacy-oriented learning. In Proceedings of the AAAI Conference on Artificial Intelligence, volume 33, pages 6618$6625,2019$.

[7] Xiang Kong, Qizhe Xie, Zihang Dai, and Eduard Hovy. Fast and simple mixture of softmaxes with bpe and hybrid-lightrnn for language generation. In Proceedings of the AAAI Conference on Artificial Intelligence, volume 33, pages 6626-6633, 2019.

[8] Minh-Thang Luong, Hieu Pham, and Christopher D Manning. Effective approaches to attention-based neural machine translation. arXiv preprint arXiv:1508.04025, 2015.

[9] Kishore Papineni, Salim Roukos, Todd Ward, and Wei-Jing Zhu. Bleu: a method for automatic evaluation of machine translation. In Proceedings of the 40th annual meeting on association for computational linguistics, pages 311-318. Association for Computational Linguistics, 2002.

[10] Rico Sennrich, Barry Haddow, and Alexandra Birch. Neural machine translation of rare words with subword units. arXiv preprint arXiv:1508.07909, 2015 .

[11] Ashish Vaswani, Noam Shazeer, Niki Parmar, Jakob Uszkoreit, Llion Jones, Aidan N Gomez, Łukasz Kaiser, and Illia Polosukhin. Attention is all you need. In Advances in Neural Information Processing Systems, pages 59986008, 2017.

[12] Zhilin Yang, Zihang Dai, Ruslan Salakhutdinov, and William W Cohen. Breaking the softmax bottleneck: A high-rank rnn language model. arXiv preprint arXiv:1711.03953, 2017. 